\title{
Metabolic syndrome in patients with prostate cancer undergoing intermittent androgen-deprivation therapy
}

\author{
Mohammadali Mohammadzadeh Rezaei, MD; Mohammadhadi Mohammadzadeh Rezaei, MD; \\ Alireza Ghoreifi, MD; Behzad Feyzzadeh Kerigh, MD
}

Department of Urology, Masshad University of Medical Sciences, Iran

Cite as: Can Urol Assoc J 2016;10(9-10):E300-5. http://dx.doi.org/10.5489/cuai.3655 Published online September 13, 2016

\section{Abstract}

Introduction: The presence of metabolic syndrome in men with prostate cancer (PCa) undergoing androgen-deprivation therapy (ADT), especially intermittent type, has not been completely evaluated. The aim of this study is to evaluate metabolic syndrome in men with PCa undergoing intermittent ADT.

Methods: In this longitudinal study, we studied the prevalence of metabolic syndrome and its components in 190 patients who were undergoing intermittent ADT. The metabolic syndrome was defined according to the Adult Treatment Panel III criteria. All metabolic parameters, including lipid profile, blood glucose, blood pressures, and waist circumferences of the patients were measured six and 12 months after treatment.

Results: Mean age of the patients was $67.5 \pm 6.74$ years. The incidence of metabolic syndrome after six and 12 months was $6.8 \%$ and $14.7 \%$, respectively. Analysis of various components of the metabolic syndrome revealed that patients had significantly higher overall prevalence of hyperglycemia, abdominal obesity, and hypertriglyceridemia in their six- and 12-month followups, but blood pressure has not been changed in the same period except for diastolic blood pressure after six months.

Conclusions: Although there was an increased risk of metabolic syndrome in patients receiving intermittent ADT, it was lower than other studies that treated the same patients with continuous ADT. Also it seems that intermittent ADT has less metabolic complications than continuous ADT and could be used as a safe alternative in patients with advanced and metastatic PCa.

\section{Introduction}

Prostate cancer (PCa) is the most common non-cutaneous cancer diagnosed in men and the second most common cause of cancer related death, with a lifetime risk of death at $2.57 \%{ }^{1,2}$ Statistics suggest that its incidence and the death rate are rapidly increasing, despite improved detection rates. This may be due to rapid increases in the population age and changes in dietary habits. ${ }^{3}$
Radical prostatectomy and radiotherapy are the preferred treatments in men with confined PCa. However, androgendeprivation therapy (ADT) is the main treatment of advanced and metastatic PCa. The modalities of ADT include surgery (orchiectomy) or medical therapy. ${ }^{4}$ Today, with progression in new medical drugs (gonadotropin-releasing hormone [GnRH] agonists and antagonists), most patients choose medical therapy, so the overall use of ADT has increased in the past two decades. Also because of the increasing rate of PCa diagnosis in younger men, hormonal therapy is being used for extended periods of time. ${ }^{5}$ Different studies showed that most men with PCa die of conditions other than their primary malignancy, so changes in treatment modalities and diagnosis and management of adverse effects have become more important. Therefore, different strategies for ADT, such as intermittent treatment have been described. Intermittent androgen suppression may reduce the side effects of therapy during off-treatment periods. ${ }^{6}$

The resulting hypogonadism due to ADT may leads to adverse results, such as osteoporosis, unfavourable body composition (increase in body mass index, increased fat mass, reduced lean body mass and muscle strength), sexual dysfunction, and reduced quality of life. Recent studies suggest male hypogonadism is an independent risk factor for the development of metabolic syndrome and diabetes. ${ }^{7}$ However, the presence of metabolic syndrome in men with PCa undergoing ADT, especially intermittent type, has not been completely evaluated. In this study, we evaluated metabolic syndrome in men with PCa undergoing intermittent ADT.

\section{Methods}

In this longitudinal study, we studied the patients who were undergoing intermittent ADT. All patients did not have metabolic syndrome at the start of study. Exclusion criteria were abnormal liver function tests or serum creatinine, history of any medical diseases (such as thyroid disease, hypogonadism, or glucocorticoid use), and any history of chemotherapy. Also, if prostate-specific antigen (PSA) and symptoms 
did not respond to a full course of ADT, the patient was considered castration-resistant and excluded from the study.

The metabolic syndrome was defined according to the Adult Treatment Panel III criteria. Patients were classified as having metabolic syndrome if three of the following five criteria were present: fasting plasma glucose level more than $110 \mathrm{mg} / \mathrm{dL}$, serum triglyceride level more than $150 \mathrm{mg} / \mathrm{dL}$, serum high-density lipoprotein (HDL) level less than $40 \mathrm{mg} /$ $\mathrm{dL}$, waist circumference more than $102 \mathrm{~cm}$, and blood pressure of more than 130/85 mmHg. Participants on antihypertensive drugs and lipid-lowering medications were classified as positive for the respective criterion.

One hundred ninety patients who were candidates for ADT and responded to six injections of $\mathrm{GnRH}$ agonists were enrolled in this study. The patients were categorized into three groups: patients with locally advanced disease who were not suitable for radical prostatectomy; patients with metastatic disease; and patients with recurrent disease following radical prostatectomy or radiotherapy. All patients signed the informed consent. Response was defined as PSA level below $4.0 \mathrm{ng} / \mathrm{L}$. During this induction therapy, all patients received injections of triptorelin $3.75 \mathrm{mg}$ every 28 days plus flutamide $250 \mathrm{mg}$ three times daily for the first two weeks. Serum PSA and testosterone, as well as clinical status, were checked every three months and if PSA rose to $10 \mathrm{ng} / \mathrm{L}$, patients received three more injections to suppress the serum PSA to $4 \mathrm{ng} / \mathrm{L}$ or less. All metabolic parameters, including lipid profile, blood glucose, blood pressures, and waist circumferences of the patients were measured six and 12 months after treatment. All data were analyzed using SPSS version 18.0 software and $p<0.05$ was considered statistically significant.

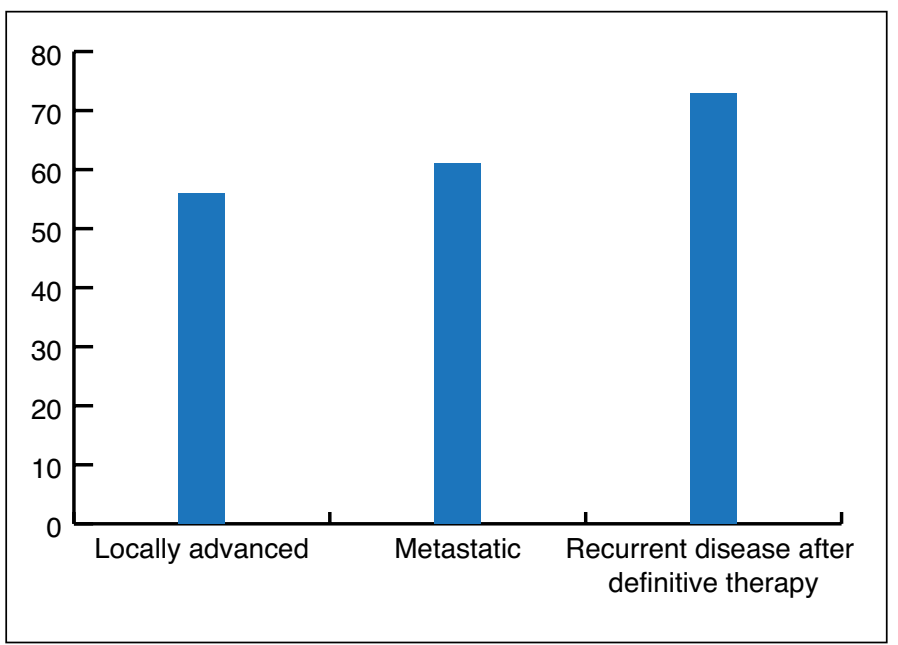

Fig. 1. Patient categories.

\section{Results}

A total of 190 patients were evaluated in this study. The mean age of the patients was $67.5 \pm 6.74$ years $(\min 58$ and max 80). Of these 190 patients, 56 patients (29.5\%) had locally advanced PCa, 61 (32.1\%) had metastatic disease, and 73 (38.4\%) had recurrent disease after previous definitive therapy (Fig. 1). Their Gleason scores (GS) were as following: GS 6 in 12 patients (6.3\%), GS 7 in 45 patients $(23.7 \%)$, GS 8 in 48 patients $(25.2 \%)$, GS 9 in 57 patients (30\%) and GS 10 in 28 patients (14.7\%) (Fig. 2).

Analysis of various components of the metabolic syndrome revealed that patients had significantly higher overall prevalence of hyperglycemia, abdominal obesity, and hypertriglyceridemia in their six- and 12-month followups, but blood pressure had not changed in the same period except for diastolic blood pressure after six months (Table 1).

Fasting plasma glucose of the patients was $111.92 \pm$ $13.74,112.78 \pm 16.4$, and $114.58 \pm 17.66 \mathrm{mg} / \mathrm{dl}$ at the beginning of the study and at six and 12 month post-treatment, respectively. The change was not significantly different in the first six month $(p=0.107)$, but after 12 months it was significantly higher $(p<0.001)$. The mean amounts of triglycerides in patients at the same periods were $104.92 \pm$ $20.92,110.82 \pm 18.58$, and $123.57 \pm 17.69 \mathrm{mg} / \mathrm{dL}$, respectively, a significant increase after both six and 12 months $(p<0.001)$. Also the mean amount of HDL cholesterol was $47.78 \pm 9.54,50.25 \pm 8.99$, and $51.09 \pm 8.18 \mathrm{mg} / \mathrm{dl}$ ), again a significant increase at six and 12 months $(p<0.001)$.

The mean waist circumferences of the patients at the start and at six- and 12-month followups was $90.37 \pm 13.3,91.04$ \pm 13.25 , and $91.41 \pm 13.67 \mathrm{~cm}$, respectively, a significant increased after six and 12 months $(p<0.001)$.

In the same time periods, the mean systolic blood pressures of the patients were $126.35 \pm 15.38,127.52 \pm 2.80$, and $127.23 \pm 12.15 \mathrm{mmHg}$, respectively. The changes were

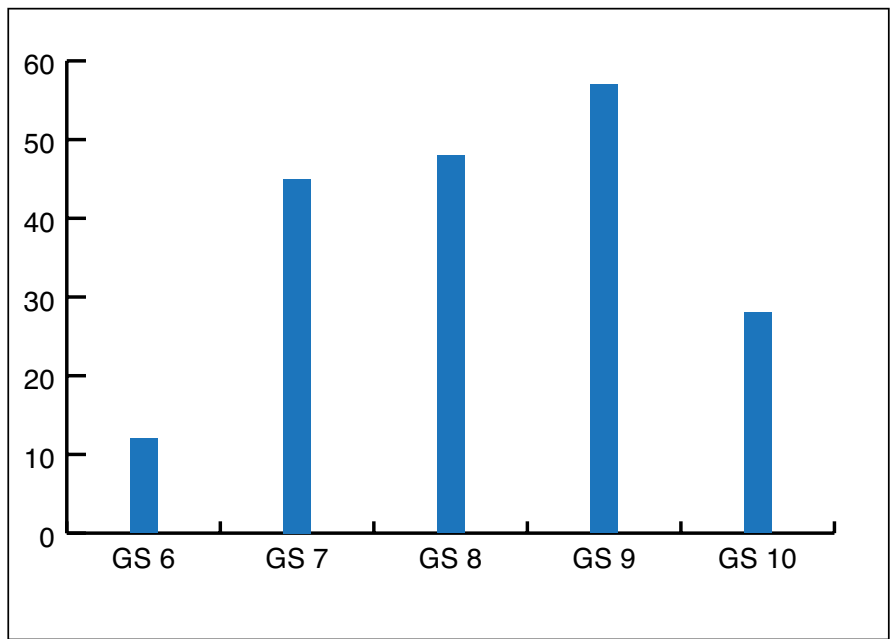

Fig. 2. Gleason scores (GS) of the prostate cancers. 


\begin{tabular}{|c|c|c|c|c|c|}
\hline \multirow{2}{*}{ Characteristic } & \multirow{2}{*}{ First of study } & \multirow{2}{*}{ After 6 months } & \multirow{2}{*}{ After 12 months } & \multicolumn{2}{|c|}{$p$ value } \\
\hline & & & & 6 months & 12 months \\
\hline Fasting plasma glucose & $111.92 \pm 13.74$ & $112.78 \pm 16.4$ & $114.58 \pm 17.66$ & 0.0107 & $<0.001$ \\
\hline Triglyceride & $104.92 \pm 20.92$ & $110.82 \pm 18.58$ & $123.57 \pm 17.69$ & $<0.001$ & $<0.001$ \\
\hline High-density lipoprotein & $47.78 \pm 9.54$ & $50.25 \pm 8.99$ & $51.09 \pm 8.18$ & $<0.001$ & $<0.001$ \\
\hline Waist circumference & $90.37 \pm 13.3$ & $91.04 \pm 13.25$ & $91.41 \pm 13.67$ & $<0.001$ & $<0.001$ \\
\hline Systolic blood pressure & $126.35 \pm 15.38$ & $127.52 \pm 12.80$ & $127.23 \pm 12.15$ & 0.098 & 0.252 \\
\hline Diastolic blood pressure & $82.21 \pm 9.47$ & $83.18 \pm 8.41$ & $81.31 \pm 8.07$ & 0.029 & 0.055 \\
\hline
\end{tabular}

not significantly different $(\mathrm{p}=0.098$ and 0.252 after six and 12 months, respectively). Also, the mean diastolic blood pressures of the patients were $82.21 \pm 9.47,83.18 \pm 8.41$, and $81.31 \pm 8.07 \mathrm{mmHG}$, respectively; while the changes were significantly different after six months $(p=0.029)$, they were not was not after 12 months $(p=0.055)$.

After six months, 13 patients $(6.8 \%)$ met the criteria of metabolic syndrome. The mean age of patients with metabolic syndrome was not statistically different that that of the other patients $(70.92 \pm 8.19$ vs. $68.35 \pm 6.61$ years; $\mathrm{p}=0.185)$. Also, the incidence of metabolic syndrome after 12 months was $14.7 \%$ (28 patients). At this time period, the mean age of patients with metabolic syndrome was significantly higher than that of the other patients $(71.21 \pm 7.09$ vs. $68.06 \pm 6.59$ years; $p=0.022$ ).

\section{Discussion}

ADT has been the cornerstone of treatment of advanced and metastatic PCa. It has progressed since 1941, when Huggins et al described the androgen dependence of PCa by showing that castration decreases its growth. ${ }^{8} \mathrm{ADT}$ includes orchidectomy or medical therapy (GnRH agonists or antagonists), but most patients choose the latter. ${ }^{4} \mathrm{GnRH}$ agonists were discovered in 1971 and are now the mainstay of PCa treatment; approximately 600000 men in the U.S. alone are receiving it. ${ }^{9}$ The overall use of ADT has increased in the past two decades and most men with PCa die of conditions other than their primary malignancy, such as ADT complications. In order to increase quality of life (QoL), different strategies for ADT, with possibly fewer complications, have been described. This includes intermittent treatment. ${ }^{5,6}$ Most phase 2 studies have shown that intermittent ADT regimens decrease adverse events and improve QoL during offtherapy periods, with promising tolerability profiles. Also, early phase 3 results show a better tolerability profile and QoL with intermittent ADT than with continuous treatment. Although these early studies demonstrate the potential shortterm benefits of intermittent ADT, there are still insufficient data to determine whether it has the potential to prevent or reverse the long-term complications associated with ADT. ${ }^{10}$

\section{ADT and metabolic syndrome}

One of the most challenging side effects of ADT is metabolic syndrome, which may be due to hypogonadism. Few studies have evaluated the metabolic alterations that occur as a result of ADT in men with PCa. Braga-Basaria et al found that more than half of the men receiving long-term (at least 12 months) ADT had metabolic syndrome compared with onefifth of the men in control groups. They suggest that the high prevalence of metabolic syndrome in the ADT group may be a direct result of profound hypogonadism and independent of any influence of age, race, and disease (PCa). ${ }^{11}$ In their study, Valverde et al showed that the prevalence of metabolic syndrome in patients on ADT was $51.9 \%$ compared to $35.8 \%$ in patients without ADT. ${ }^{12}$ In a cross-section observational study, Munoz Garcia et al stated that prevalence of metabolic syndrome in men on ADT after six months of treatment is $21 \%$, after $12-18$ months treatment is $36 \%$, and after 24 months treatment is $24 \% .^{13}$ Finally, in a recent metaanalysis, there was a positive association between ADT and risk of metabolic syndrome (relative risk 1.75$).{ }^{14}$ In our study, the prevalence of metabolic syndrome in patients treated with intermittent ADT was $6.8 \%$ and $14.7 \%$ after six and 12 months, respectively. Although there was an increased risk of metabolic syndrome in these patients, this increase was lower than other studies that treated the same patients with continuous ADT. It seems that the risk of metabolic syndrome in intermittent ADT is lower than with continuous treatment.

\section{ADT and diabetes}

There are some studies that have evaluated the components of metabolic syndrome in patients treated with ADT. In the meta-analysis mentioned above, diabetes was the only component present in more than three of nine studies, and also showed an increased risk following ADT (relative risk 1.36). ${ }^{14}$ Keating et al, in an observational study on 37 443 population-based men who were diagnosed with local or regional PCa, showed that treatment with GnRH agonists was associated with statistically significantly increased risk of incident diabetes (adjusted hazard ratio 1.44). They showed 159.4 events of diabetes per 1000 person-years 
in patients on ADT compared to 87.5 events for no ADT patients. ${ }^{15}$ In their study, Lage et al showed that the estimated relative risk of incident diabetes associated with the receipt of ADT was $1.36 .{ }^{16}$ Basaria et al also showed that men on ADT had significantly higher levels of fasting serum glucose compared with men not on ADT. In their study, $44 \%$ of ADT patients had glucose levels in the diabetic range and the duration of ADT was directly related to the severity of these metabolic abnormalities. ${ }^{17}$ Alibhai et al, in an age-matched cohort study, showed that men on ADT who were followed for an average of 6.47 years had an increased risk of diabetes compared with men who did not receive ADT. ${ }^{18}$ A recent study on Chinese patients who were given ADT showed a higher risk of diabetes mellitus (hazard ratio 4.39). ${ }^{19}$ In the Barga-Basiria study, hyperglycemia and abdominal obesity were the major determinants of the higher prevalence of metabolic syndrome in this group, leading to the conclusion that hyperinsulinemia in these patients was associated with increase in fat mass. ${ }^{11}$ On the other hand, some short-term studies have shown different results. Smith et al, in a short-term prospective study of 22 men with PCa undergoing ADT, showed a significant increase in insulin levels after three months of treatment compared with baseline; however, there was no significant change in plasma glucose levels. ${ }^{20}$ In their study, Dockery et al showed that ADT for three months resulted in a $63 \%$ increase in fasting insulin levels without any changes in fasting glucose. ${ }^{21} \mathrm{~A}$ study done by Nishiyama et al evaluated 49 men with PCa undergoing ADT for six months showed a mild increase in fasting blood glucose (from 103-106 mg/dl), but the levels remained below the diabetes range. ${ }^{22}$ In a recent study, Davis et al retrospectively reviewed the charts of 100 consecutive men with intermediate or high-risk localized PCa referred to the British Columbia Cancer Agency for ADT; baseline diabetes or impaired glucose tolerance was $24 \%$, but in study cohort, they had a similar prevalence (odds ratio $0.93 ; p=0.8$ ). ${ }^{23}$ According to the various studies mentioned above, the effect of ADT on plasma glucose is different in short- and long-term followup. These studies suggest an increased level of insulin develops within a few months of starting ADT and prevents the development of hyperglycemia. The etiology of this hyperinsulinemia is not clearly defined. There is some evidence that testosterone can modulate hepatic and lipoprotein lipases in visceral adipose tissue, which consequently affects insulin clearance. ${ }^{24}$ However, it seems that in the long-term, male hypogonadism is associated with a decrease in lean body mass (LBM) and an increase in fat mass, which results in elevated levels of adiponektines, which in turn, are responsible for causing insulin resistance. ${ }^{25}$ In our study, the change of fasting blood glucose was not significantly different in the first six months, but after 12 months it was significantly higher. Our results showed that in short-term treatment with intermittent ADT there is no difference in fasting blood glucose, which is in agreement with other short-term studies. However, in longterm followup, there is significant hyperglycemia.

\section{ADT and hyperlipidemia}

In their study, Davis et al stated that baseline dyslipidemia was present in $51 \%$ patients receiving ADT. ${ }^{23}$ In a study by Bo et al, after three months, ADT group had increased levels of low-density lipoprotein ( $\mathrm{LDL}$ ) compared to the control groups. After 12 months, ADT group had increased levels of waist circumference, total cholesterol, HDL, and LDL compared to the other two groups. ${ }^{26}$ Dockery et al, in their study, showed that continuous ADT for three months resulted in increased levels of cholesterol and HDL, but no significant change occurred in LDL or triglyceride levels. ${ }^{21}$ In their study, Eri et al evaluated the impact of the luteinizing hormone-releasing hormone (LHRH) agonist leuprolide on 26 patients with benign prostatic hyperplasia (BPH) and showed that the mean total cholesterol level increased by $10.6 \%$, HDL cholesterol by $8.2 \%$, and triglycerides by $26.9 \%$, but LDL levels were unchanged. ${ }^{27}$ Smith et al in their study on 26 men treated with leuprolide for 12 months showed the same increase in HDL levels. ${ }^{28}$ In our study, the mean amount of trigycerides in patients significantly increased after both six and 12 months. Also, the mean amounts of HDL cholesterol and the mean waist circumferences significantly increased after the same periods. Hyperinsulinemia might have been expected to cause a decrease in HDL levels, therefore, some other non-investigated factors may be involved in the complex relationship between androgens and metabolic factors. Maybe an increase in adiponektin levels due to an increase in fat mass in these patients contributed to high levels of cholesterol (especially HDL).

\section{ADT and hypertension}

The role of androgens in cardiovascular disease (CVD) in men has long been debated. Previous studies mainly evaluated the relationship between ADT and CVD, but few of them focused on the changes in blood pressure and arterial compliance. Some epidemiologic studies have shown that low testosterone levels in men are independently associated with hypertension. A recent study in patients with PCa undergoing short-term ADT showed a significant decrease in systemic arterial compliance. ${ }^{21}$ On the other hand, Smith et al showed no significant change in blood pressure after 12 months treatment with leuprolide. ${ }^{28}$ Davis et al showed that hypertension was more prevalent in ADT patients than in an age- and sex-matched population sample (odds ratio $1.74 ; \mathrm{p}=0.006) .{ }^{23}$

Previous studies have shown that approximately half of men with PCa die of non-cancer-related causes, and the 
most common etiology is CVD. ${ }^{11}$ Keating et al showed an association between ADT and CVD (adjusted hazard ratio 1.16), myocardial infarction (hazard ratio 1.11), and sudden cardiac death or life-threatening ventricular arrhythmia (hazard ratio 1.16). ${ }^{29}$ Another study by Tsai et al also showed that men receiving ADT were 2.6 times more likely to die of $\mathrm{CV}$ events than non-ADT controls. They found that men who received ADT had a $20 \%$ higher risk of serious CV morbidity than similar men who did not. ${ }^{30}$ Although these studies showed a relationship between ADT and increased risk of CVD, some other studies have not confirmed this association. Alibhai et al, in a cohort study of nearly 20000 ADT users who were observed for a mean of 6.47 years, showed an increased risk of diabetes, but there was no such association with incidence of acute myocardial infarction. ${ }^{18}$ An analysis from the Radiation Therapy Oncology Group showed similar results. The study found that long-term ADT does not increase $\mathrm{CV}$ mortality in men with locally advanced PCa. ${ }^{31}$ A recent, large study assessing the impact of ADT on CVD mortality in men undergoing curative-intent external beam radiation therapy confirmed these findings. Among these men, the cumulative CV mortality at seven years was $2.6 \%$ for men not undergoing ADT, 2.1\% for men with six months ADT, and $1.4 \%$ for men undergoing ADT for six months. ${ }^{32}$ Punnen et al also analyzed a total of 7248 men to assess the relationship between ADT and CV mortality and found that there was no significant difference in CV mortality among men who did vs. those who did not receive ADT. ${ }^{33}$

Although the relationship between ADT and CVD is controversial, many authors have shown that the profound hypogonadism in patients undergoing ADT may be responsible for high CV mortality. Low testosterone levels are a risk factor for metabolic syndrome and metabolic syndrome is also a known cause of increased CV mortality. Men with metabolic syndrome are three times more likely to die of coronary heart disease and other CVD. ${ }^{11}$ Still, some have voiced concerned that unmeasured factors maybe confounding the relationship between ADT use and CV mortality. ${ }^{33}$ In our study, the changes in mean systolic blood pressures of the patients were not significantly different after six and 12 months. On the other hand, the changes in mean diastolic blood pressures of the patients were significantly different after six months, but not after 12 months. According to CV changes, it would appear intermittent ADT is safer than continuous ADT. Long-term studies are needed to document the effect of intermittent ADT on morbidity and mortality due to CVD.

To our knowledge, this study is the first study that evaluates the metabolic syndrome in patients receiving intermittent type of ADT. The major limitation of our study is that it was an observational study, so we recommend a long-term, prospective study with a larger sample size.

\section{Conclusion}

Our study showed that although there was an increased risk of metabolic syndrome in patients receiving intermittent ADT, this increase was lower than other studies that treated the same patients with continuous ADT. We concluded that the risk of metabolic syndrome in intermittent ADT is lower than that with continuous treatment. Further, patients on ADT had significantly higher overall prevalence of hyperglycemia, abdominal obesity, and hypertriglyceridemia, but blood pressure did not change in the 12-month follow up (except for diastolic blood pressure after six months). We therefore further concluded that intermittent ADT has less metabolic complications than continuous ADT and could be used as a safe alternative in patients with advanced and metastatic PCa.

Competing interests: The authors report no competing personal or financial interests.

This paper has been peer-reviewed.

\section{References}

1. Siegel R, Ward E, Brawley 0, et al. Cancer statistics 2011. CA Cancer J Clin 2011;61:212-36. http:// dx.doi.org/10.3322/caac.20121

2. Shahab AA, Soebadi DM, Djatisoesanto W, et al. Prostate-specific antigen and prostate-specific antigen density cutoff points among Indonesian population suspected for prostate cancer. Prostate Int 2013;1:2330. http://dx.doi.org/10.12954/PI.12003

3. Han HH, Park JW, Na JC, et al. Epidemiology of prostate cancer in South Korea. Prostate Int 2015;3:99102. http://dx.doi.org/10.1016/i.prnil.2015.06.003

4. Perlmutter MA, Lepor H. Androgen-deprivation therapy in the treatment of advanced prostate cancer. Rev Urol 2007;9Suppl1:S3-8.

5. Shahinian VB, Kuo YF, Freeman JL, et al. Increasing use of gonadotropin-releasing hormone agonists for the treatment of localized prostate carcinoma. Cancer 2005;103:1615-24. http://dx.doi.org/10.1002/ cncr. 20955

6. Tunn UW, Canepa G, Kochanowsky A, et al. Testosterone recovery in the off-treatment time in prostate cancer patients undergoing intermittent androgen deprivation therapy. Prostate Cancer Prostatic Dis 2012;15:296-302. http://dx.doi.org/10.1038/pcan.2012.12

7. Basaria $S$, Lieb J 2nd, Tang AM, et al. Long-term effects of androgen-deprivation therapy in prostate cancer patients. Clin Endocrinol (Oxf) 2002;56:779-86. http://dx.doi.org/10.1046/i.13652265.2002.01551.x

8. Hoimes CJ, Kelly WK. Redefining hormone resistance in prostate cancer. Ther Adv Med Oncol 2010;2:10723. http://dx.doi.org/10.1177/1758834009356433

9. Lepor H, Shore ND. LHRH agonists for the treatment of prostate cancer: 2012. Rev Urol 2012;14:1-12.

10. Abrahamsson PA. Potential benefits of intermittent androgen suppression therapy in the treatment of prostate cancer: A systematic review of the literature. Eur Urol 2010;57:49-59. http://dx.doi. org/10.1016/i.eururo.2009.07.049

11. Braga-Basaria M, Dobs AS, Muller DC, et al. Metabolic syndrome in men with prostate cancer undergoing long-term androgen-deprivation therapy. J Clin Oncol 2006;24:3979-83. http://dx.doi.org/10.1200/ JC0.2006.05.9741

12. Valverde J, Planas Morin J, Salvador Lacambra C, et al. Prevalence of metabolic syndrome in prostate cancer patients under androgen-deprivation therapy: Interim results of a case-control study. Eur Urol Suppl 2011;10:337. http://dx.doi.org/10.1016/S1569-9056(11)61074-9

13. Munoz Garcia J, Samper Ots P, Rios Kavadoy Y, et al. Cross-section observational study about prevalence of metabolic syndrome and osteoporosis in prostate cancer treated with androgen-deprivation therapy and their impact on quality of life (SIMBOSPROST): Gicor Group. Intl J Rad Onc Bio Phys 2013; 87:S356. http://dx.doi.org/10.1016/i.i.iobp.2013.06.934 
14. Bosco C, Crawley D, Adolfsson J, et al. Quantifying the evidence for the risk of metabolic syndrome and its components following androgen-deprivation therapy for prostate cancer: A meta-analysis. PLoS One 2015;10:e01 17344. http://dx.doi.org/10.1371/journal.pone.0117344

15. Keating NL, $O^{\prime}$ Malley AJ, Freedland SJ, et al. Diabetes and cardiovascular disease during androgendeprivation therapy: Observational study of veterans with prostate cancer. J Natl Cancer Inst 2010;102:3946. http://dx.doi.org/10.1093/inci/dip404

16. Lage MJ, Barber BL, Markus RA. Association between androgen-deprivation therapy and incidence of diabetes among males with prostate cancer. Urology 2007;70:1104-8. http://dx.doi.org/10.1016/i. urology.2007.08.012

17. Basaria S, Muller DC, Carducci MA, et al. Hyperglycemia and insulin resistance in men with prostate carcinoma who receive androgen-deprivation therapy. Cancer 2006;106:581-8. http://dx.doi.org/10.1002/ cncr.21642

18. Alibhai $S M$, Duong-Hua $M$, Sutradhar $R$, et al. Impact of androgen-deprivation therapy on cardiovascular disease and diabetes. J Clin Oncol 2009;27:3452-8 http://dx.doi.org/10.1200/JC0.2008.20.0923

19. Teoh JY, Chiu PK, Chan SY, et al. Androgen-deprivation therapy, diabetes and poor physical performance status increase fracture risk in Chinese men treated for prostate cancer. Aging Male 2015;18:180-5. http://dx.doi.org/10.3109/13685538.2015.1046043

20. Smith JC, Bennett $S$, Evans $L M$, et al. The effects of induced hypogonadism on arterial stiffness, body composition, and metabolic parameters in males with prostate cancer. J Clin Endocrinol Metab 2001;86:42617. http://dx.doi.org/10.1210/icem.86.9.7851

21. Dockery F, Bulpitt CJ, Agarwal S, et al. Testosterone suppression in men with prostate cancer leads to an increase in arterial stiffness and hyperinsulinaemia. Clin Sci (Lond) 2003;104:195-201. http:// dx.doi.org/10.1042/cs1040195

22. Nishiyama $\mathrm{T}$, Ishizaki F, Tsutomu A, et al. The influence of androgen-deprivation therapy on metabolism in patients with prostate cancer. J Clin Endocrinol Metab 2005;90:657-60. http://dx.doi.org/10.1210/ ic.2004-1611

23. Davis MK, Rajala JL, Tyldesley S, et al. The prevalence of cardiac risk factors in men with localized prostate cancer undergoing androgen-deprivation therapy in British Columbia, Canada. J Oncol 2015;2015:820403. http://dx.doi.org/10.1155/2015/820403

24. Pugeat $M$, Moulin $P$, Cousin $P$, et al. Interrelations between sex hormone-binding globulin (SHBG), plasma lipoproteins, and cardiovascular risk. I Steroid Biochem Mol Biol 1995;53:567-72. http:// dx.doi.org/10.1016/0960-0760(95)00102-6
25. Geer $\mathrm{EB}$, Shen W. Gender differences in insulin resistance, body composition, and energy balance. Gend Med 2009;6Suppl1:60-75. http://dx.doi.org/10.1016/i.genm.2009.02.002

26. Bo JJ, Zhang C, Zhang LH, et al. Androgen-deprivation therapy through bilateral orchiectomy: Increased metabolic risks. Asian J Androl 2011;13:833-7. http://dx.doi.org/10.1038/aja.2011.83

27. Eri LM, Urdal P, Bechensteen AG. Effects of the luteinizing hormone releasing hormone agonist leuprolide on lipoproteins, fibrinogen and plasminogen activator inhibitor in patients with benign prostatic hyperplasia. J Urol 1995;154:100-4. htrp://dx.doi.org/10.1016/S0022-5347(01)67239-2

28. Smith MR, Lee H, McGovern F, et al. Metabolic changes during gonadotropin-releasing hormone agonist therapy for prostate cancer: Differences from the classic metabolic syndrome. Cancer 2008; 112:2188-94. http://dx.doi.org/10.1002/cncr.23440

29. Keating NL, $O^{\prime}$ Malley AJ, Smith MR. Diabetes and cardiovascular disease during androgen-deprivation therapy for prostate cancer. J Clin Oncol 2006;24:4448-56. http://dx.doi.org/10.1200/JC0.2006.06.2497

30. Tsai HK, D'Amico AV, Sadetsky N, et al. Androgen-deprivation therapy for localized prostate cancer and the risk of cardiovascular mortality. J Natl Cancer Inst 2007;99:1516-24. http://dx.doi.org/10.1093/ inci/dim 168

31. Efstathiou JA, Bae K, Shipley WU, et al. Cardiovascular mortality and duration of androgen-deprivation for locally advanced prostate cancer: Analysis of RTOG 92-02. Eur Urol 2008;54:816-23. http://dx.doi. org/10.1016/i.eururo.2008.01.021

32. Kim J, Vaid M, Tyldesley S, et al. Population-based study of cardiovascular mortality among patients with prostate cancer treated with radical external bean radiation therapy with and without adjuvant androgendeprivation therapy at the Birtish Columbia Cancer Agency. Int J Radiat Oncol Biol Phys 2011;80:742-50. http://dx.doi.org/10.1016/i.ijrobp.2010.03.018

33. Punnen S, Cooperberg MR, Sadetsky N, et al. Androgen-deprivation therapy and cardiovascular risk. J Clin Oncol 2011;29:3510-6. http://dx.doi.org/10.1200/JC0.2011.35.1494

Correspondence: Dr. Alireza Ghoreifi, Department of Urology, Masshad University of Medical Sciences, Iran; aghoreifi@yahoo.com 